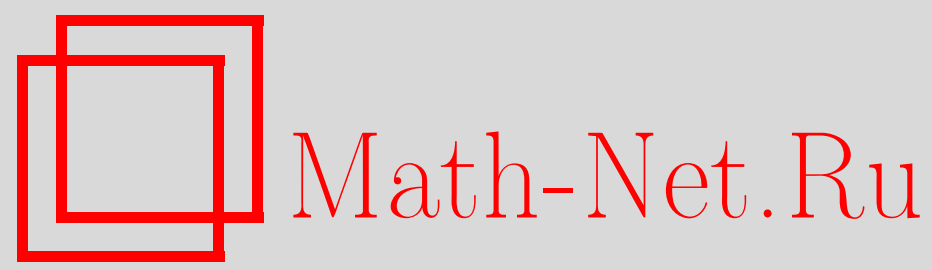

И. Д. Кан, Представление чисел линейными формами, $M a$ тем. заметки, 2000, том 68, выпуск 2, 210-216

DOI: https://doi.org/10.4213/mzm939

Использование Общероссийского математического портала Math-Net.Ru подразумевает, что вы прочитали и согласны с пользовательским соглашением http://www . mathnet.ru/rus/agreement

Параметры загрузки:

IP : 54.237 .206 .68

26 апреля 2023 г., 16:21:38

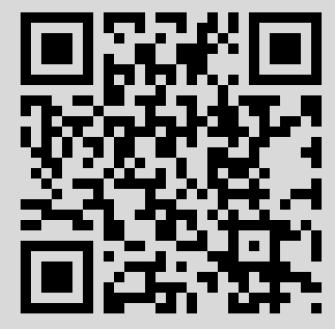


УДК 511.2

\section{ПРЕДСТАВЛЕНИЕ ЧИСЕЛ ЛИНЕЙНЫМИ ФОРМАМИ}

\section{И. Д. Кан}

В статье доказаны новые теоремы по проблеме Фробениуса, относящейся к аддитивной теории чисел, с помощью цепных дробей.

Библиографоия: 12 названий.

1. Введение. Говорят, что целое число $N$ представимо через натуральные числа $a_{0}, a_{1}, \ldots, a_{m}$, если существуют целые неотрицательные числа $x_{0}, x_{1}, \ldots, x_{m}$ такие, что $N=a_{0} x_{0}+a_{1} x_{1}+\cdots+a_{m} x_{m}$. Множество чисел $N$, представимых через $a_{0}, a_{1}, \ldots, a_{m}$, обозначим через $\left\langle a_{0}, a_{1}, \ldots, a_{m}\right\rangle$.

Проблема Фробениуса состоит в нахождении наибольшего целого числа $g\left(a_{0}, a_{1}, \ldots\right.$, $\left.a_{m}\right)$, не представимого через взаимно простые (в совокупности) числа $a_{0}, a_{1}, \ldots, a_{m}$. Функция $g\left(a_{0}, a_{1}, \ldots, a_{m}\right)$ назьвается функиией Фробениуса. Таким образом,

$$
g\left(a_{0}, a_{1}, \ldots, a_{m}\right)=\max \left(\mathbb{Z} \backslash\left\langle a_{0}, a_{1}, \ldots, a_{m}\right\rangle\right)
$$

При $m=1$ известен классический результат Сильвестра [1]:

$$
g\left(a_{0}, a_{1}\right)=a_{0} a_{1}-a_{0}-a_{1}
$$

При $m=2$ проблему Фробениуса решил в 1960 г. Джонсон [2], однако его решение представляет собой не формулу, а лишь некоторьй “быстрьй", точнее, полиномиальный, алгоритм (определение есть в [3]). Другой алгоритм для $g\left(a_{0}, a_{1}, a_{2}\right)$ предложили в 1962 г. Брауэр и Шокли [4], получив также формулу для частного случая $a_{0}+a_{2} \equiv 0\left(\bmod a_{1}\right)$, где $a_{1}=\min \left\{a_{0}, a_{1}, a_{2}\right\}$ :

$$
g\left(a_{0}, a_{1}, a_{2}\right)=\max \left\{a_{2}\left[\frac{a_{1} a_{0}}{a_{0}+a_{2}}\right], a_{0}\left[\frac{a_{1} a_{2}}{a_{0}+a_{2}}\right]\right\}-a_{1}
$$

где $[x]$ - целая часть числа $x \in \mathbb{R}$ (здесь и в дальнейшем). Ни для какого значения $m \geqslant 3$ полиномиального алгоритма решения проблемы Фробениуса не найдено.

Работа выполнена при частичной финансовой поддержке Российского фонда фундаментальных исследований, грант № 96-01-00492. 
Тем не менее, существует ряд результатов по проблеме Фробениуса при определенных соотношений на аргументы. Так, Роберц [5] в 1956 г. нашел формулу для функции Фробениуса от арифметической прогрессии

$$
g(a, a+d, \ldots, a+m d)=a\left[\frac{a-2}{m}\right]+d(a-1),
$$

обобщив результат Брауэра [6] для $d=1$. Результат Роберца, в свою очередь, был обобщен, во-первых, Сиерингом [7], вычислившим функцию Фробениуса от объединения нескольких арифметических прогрессий с одинаковой разностью, и, во-вторых, Рёдсетом [8], решившим проблему $\Phi$ робениуса для совокупности аргументов, получаемой добавлением к арифметической прогрессии одного произвольного элемента.

Обрывая алгоритм Рёдсета на одном или нескольких первых шагах, можно получать точные формулы для функции Фробениуса, скажем, трех аргументов [9], на тем более широкой области их значений, чем большее число шагов алгоритма взято. Другой путь построения точных формул, иначе связанньй с алгоритмом Рёдсета, состоит в следующем.

Решение Рёдсета опирается на некоторые последовательности натуральных чисел, обладающие следующим свойством: каждый их член, за исключением первого и последнего, является делителем суммы своих соседей (например, арифметическая прогрессия). Все такие последовательности будем назьвать иепным.и. В [10], [11] было предложено перенести цепные последовательности из средств вычисления функции $Ф$ робениуса в множество ее аргументов с целью получения новых результатов по проблеме. Настоящая работа содержит дальнейшее развитие этого подхода.

2. Обозначения и терминология. Всюду в дальнейшем

$$
a_{n}, a_{-n+1}, \ldots, a_{-1}, a_{0}, a_{1}, \ldots, a_{m}
$$

- цепная последовательность $\left(m \geqslant 1, n \geqslant 1\right.$, НОД $\left.\left(a_{0}, a_{1}\right)=1\right)$. Это означает, что все числа $l_{j}$, где

$$
l_{j}=\frac{a_{j-1}+a_{j+1}}{a_{j}} \quad(j=-n+1,-n+2, \ldots, 0,1, \ldots, m-1)
$$

- натуральные. Числа $\varepsilon, \varepsilon_{1}, \varepsilon_{2}$ определены равенствами

$$
\varepsilon=\frac{1}{l_{1}-\frac{1}{l_{2}-\frac{1}{\ddots \cdot-\frac{1}{l_{m-1}}}}}, \quad \varepsilon_{1}=\frac{1}{l_{-1}-\frac{1}{l_{-2}-\frac{1}{\ddots \cdot-\frac{1}{l_{-n+1}}}}}, \quad \varepsilon_{2}=l_{0} 0-\varepsilon_{1} .
$$

(При этом для $m=1, n=1$ считаем, что соответственно $\varepsilon=0, \varepsilon_{1}=0$.)

\section{3. Основные результаты.}

Теорема 1. Если $a_{0}=\min \left\{a_{0}, a_{1}, \ldots, a_{m}\right\}$, то справедливо равенство

$$
g\left(a_{0}, a_{1}, \ldots, a_{m}\right)=a_{0} a_{1}-a_{0}-a_{1}-a_{0}\left[\varepsilon\left(a_{0}-1\right)\right] .
$$


ТЕорема 2. Если $a_{0}=\min \left\{a_{-n}, a_{-n+1}, \ldots, a_{0}, a_{1}, \ldots, a_{m}\right\}$, то справедливо равенство

$$
\begin{gathered}
g\left(a_{-n}, a_{-n+1}, \ldots, a_{-1}, a_{0}, a_{1}, \ldots, a_{m}\right) \\
=-a_{0}+\max \left\{a_{1}\left[\frac{a_{0} \varepsilon_{2}-a_{1}}{\varepsilon_{2}-\varepsilon}\right]-a_{0}\left[\varepsilon\left[\frac{a_{0} \varepsilon_{2}-a_{1}}{\varepsilon_{2}-\varepsilon}\right]\right],\right. \\
\left.-a_{1}\left[\frac{a_{1}-\varepsilon a_{0}}{\varepsilon_{2}-\varepsilon}\right]-a_{0}\left[-\varepsilon_{2}\left[\frac{a_{1}-\varepsilon a_{0}}{\varepsilon_{2}-\varepsilon}\right]\right]\right\} .
\end{gathered}
$$

ЗАмЕчАнИЕ 1. Частньми случаями равенств (4) и (5) теорем 1,2 являются формулы $(1)-(3)$.

ЗАмЕчАниЕ 2. Не ограничивая общности, можно (и будем в дальнейшем) считать, что $l_{j} \geqslant 2$ для всех $j$.

4. Леммы. На множестве пар целых чисел введем линейную форму $\varphi(y, z)$ по правилу $\varphi(y, z)=\left(a_{1}-a_{0}\right) y+a_{0} z$.

Напомним, что числа $a_{0}$ и $a_{1}$ по условию взаимно просты.

ЛЕмма 1. Для любого $x \in \mathbb{Z}$ существует, причем единственная, пара иелых чисел $(e(x), f(x))$ таких, что выполнены одновременно равенство $x=\varphi(e(x), f(x))$ и неравенство $0 \leqslant e(x) \leqslant a_{0}-1$.

ДокАЗАТЕЛЬСТво леммы оставляем читателю. Оно может рассматриваться как устное упражнение на полную систему вычетов и в той или иной форме содержится почти во всех учебниках по основам теории чисел.

Таким образом, функции $e(x)$ и $f(x)$ корректно определены.

На множестве пар целых чисел введем линейную форму $\theta(y, z)$ по правилу $\theta(y, z)=$ $(1-\varepsilon) y-z$.

ЛЕмма 2. Имеет место неравенство $\theta\left(a_{0}, a_{0}-a_{1}\right)>0$.

ДокАЗАТЕЛЬСТво. Для $j=0,1, \ldots, m$ через $f_{j} / e_{j}$ обозначим $j$-ю подходящую дробь к цепной дроби $1-\varepsilon$. Другими словами,

$$
\begin{array}{ll}
e_{0}=0, & e_{1}=1, \quad e_{j+1}=l_{j} e_{j}-e_{j-1}, \\
f_{0}=1, & f_{1}=1, \quad f_{j+1}=l_{j} f_{j}-f_{j-1} .
\end{array}
$$

Равенство

$$
a_{j}=\varphi\left(e_{j}, f_{j}\right)=\left(a_{1}-a_{0}\right) e_{j}+a_{0} f_{j} \quad(j=0,1, \ldots, m)
$$

проверяется непосредственной индукцией. В частности,

$$
0<a_{m}=\left(a_{1}-a_{0}\right) e_{m}+a_{0} f_{m}
$$

Поскольку $1-\varepsilon=f_{m} / e_{m}$, то из (7) следует, что $\theta\left(a_{0}, a_{0}-a_{1}\right)=(1-\varepsilon) a_{0}+a_{1}-a_{0}=$ $a_{m} / e_{m}>0$. Лемма доказана.

Определим функцию $\theta(x)$ целого аргумента $x$ по правилу $\theta(x)=\theta(e(x), f(x))$. 
Лемма 3. $Е с л и \varphi(y, z)=x$ u $y \geqslant 0, m o \theta(x) \leqslant \theta(y, z)$.

ДокАЗАТЕЛЬСТво. Пусть в условиях леммы для некоторого целого $y_{1}$ выполнены неравенства $0 \leqslant y_{1} \leqslant a_{0}$ и $y_{1} \neq y$. Пусть, кроме того, выполнено равенство $\varphi\left(y_{1}, z_{1}\right)=$ $x=\varphi(y, z)$. Тогда из леммы 1 следует, что $y \geqslant a_{0}>y_{1}$. Поскольку $\varphi\left(y-y_{1}, z-z_{1}\right)=0$, то, полагая $y_{1}=e(x)$ и $z_{1}=f(x)$, имеем:

$$
\theta(x)=\theta\left(y_{1}, z_{1}\right)=\theta(y, z)-\frac{y-y_{1}}{a_{0}} \theta\left(a_{0}, a_{0}-1\right)<\theta(y, z),
$$

так как последнее неравенство в этой цепочке справедливо по лемме 2. Лемма доказана.

ЛЕмма 4. Если $x$ представимо через $a_{0}, a_{1}, \ldots, a_{m}$, mо $\theta(x) \leqslant 0$.

ДокАЗАТЕЛЬСтво. Пусть для целых неотрицательных $k_{0}, k_{1}, \ldots, k_{m}$ выполнено равенство $x=k_{0} a_{0}+k_{1} a_{1}+\cdots+k_{m} a_{m}$. Тогда ввиду равенства (6)

$$
x=\varphi\left(k_{0} e_{0}+k_{1} e_{1}+\cdots+k_{m} e_{m}, k_{0} f_{0}+k_{1} f_{1}+\cdots+k_{m} f_{m}\right) .
$$

Поэтому в силу леммы 3

$$
\begin{aligned}
\theta(x) & =\theta\left(k_{0} e_{0}+k_{1} e_{1}+\cdots+k_{m} e_{m}, k_{0} f_{0}+k_{1} f_{1}+\cdots+k_{m} f_{m}\right) \\
& =k_{0} \theta\left(e_{0}, f_{0}\right)+\sum_{j=1}^{m} k_{j} \theta\left(e_{j}, f_{j}\right)=-k_{0}+\sum_{j=1}^{m} k_{j} e_{j}\left((1-\varepsilon)-\frac{f_{j}}{e_{j}}\right) \leqslant 0
\end{aligned}
$$

по свойству убывания подходящих дробей $f_{j} / e_{j}$ к цепной дроби $1-\varepsilon$ (см. [12]). Лемма доказана.

Лемма 5. Если $\theta(x) \leqslant 0$ и $x$ не делится на $a_{0}$, то для некоторого $j(1 \leqslant j \leqslant m)$ число х представимо через $a_{j-1}$ и $a_{j}$.

ДоказАТЕльство. Из неравенства $\theta(x) \leqslant 0$ следует, что $1-\varepsilon \leqslant f(x) / e(x)$. Пользуясь свойством убывания подходящих дробей к дроби $1-\varepsilon$, найдем $j$ такое, что

$$
1-\varepsilon \leqslant \frac{f_{j}}{e_{j}} \leqslant \frac{f(x)}{e(x)}<\frac{f_{j-1}}{e_{j-1}} .
$$

Так как $f_{j-1} e_{j}-f_{j} e_{j-1}=1$ (см. [12]), то ввиду леммы 1

$$
x=1 \cdot x=\left[\begin{array}{cc}
-f_{j} & e_{j} \\
-f_{j-1} & e_{j-1}
\end{array}\right] \cdot\left[\begin{array}{cc}
e(x) & -a_{0} \\
f(x) & a_{1}-a_{0}
\end{array}\right] .
$$

Применяя к последнему выражению правило умножения определителей (“строка на столбец"), имеем

$$
x=\left[\begin{array}{cc}
-f_{j} e(x)+e_{j} f(x) & a_{0} f_{j}+\left(a_{1}-a_{0}\right) e_{j} \\
-f_{j-1} e(x)+e_{j-1} f(x) & a_{0} f_{j-1}+\left(a_{1}-a_{0}\right) e_{j-1}
\end{array}\right] .
$$

В последнем равенстве правый столбец определителя ввиду равенства (6) состоит из чисел $a_{j}$ и $a_{j-1}$. В левом столбце того же определителя ввиду неравенства (8) верхнее число неотрищательно, нижнее - отрицательно. Вьчисляя этот определитель, получаем, что $x$ представимо через $a_{j-1}$ и $a_{j}$. Лемма доказана. 
Лемма 6. Если $a_{1}>a_{0}$ и для $\alpha \in \mathbb{R}$ выполнена оценка $0 \leqslant \alpha<a_{0}$, то справедливо равенство

$$
\max _{\substack{x \notin\left\langle a_{0}, a_{1}, \ldots, a_{m}\right\rangle \\ e(x) \leqslant \alpha}} x=a_{1}[\alpha]-a_{0}[\varepsilon[\alpha]]-a_{0} .
$$

ДокАЗАТЕльство. Так как $e(x) \leqslant[\alpha]$, то из леммы 5 и определения $\theta(x)$ следует, что для $x \notin\left\langle a_{0}, a_{1}, \ldots, a_{m}\right\rangle$ верна оценка $f(x) \leqslant-[(\varepsilon-1) e(x)]-1 \leqslant-[(\varepsilon-1)[\alpha]]-1$, следовательно,

$$
x=\left(a_{1}-a_{0}\right) e(x)+a_{0} f(x) \leqslant[\alpha]\left(a_{1}-a_{0}\right)-a_{0}([(\varepsilon-1)[\alpha]]+1) .
$$

(Ясно, что правые части равенства (9) и неравенства (10) совпадают.) Обратно: пусть $x=[\alpha]\left(a_{1}-a_{0}\right)-a_{0}([(\varepsilon-1)[\alpha]]+1)$, тогда $\theta(x)=(1-\varepsilon)[\alpha]=[(\varepsilon-1)[\alpha]]+1>0$, откуда по лемме $4 x \notin\left\langle a_{0}, a_{1}, \ldots, a_{m}\right\rangle$. Лемма доказана.

Аналогично функциям $e(x)$ и $f(x)$, определенным числами $a_{0}$ и $a_{1}$, определим функции $e_{1}(x)$ и $f_{1}(x)$ числами $a_{0}$ и $a_{-1}$ (см. начало п. 4 и лемму 1 ). Для любого целого $x$ имеем, таким образом:

$$
x=\left(a_{-1}-a_{0}\right) e_{1}(x)+a_{0} f_{1}(x), \quad 0 \leqslant e_{1}(x) \leqslant a_{0}-1 .
$$

ЛЕмма 7. Для любого иелого $x$, не кратного числу $a_{0}$, имеют место равенства

$$
e(x)=a_{0}-e_{1}(x), \quad f(x)=f_{1}(x)+\left(l_{0}-2\right) e_{1}(x)-a_{1}+a_{0} .
$$

ДокАЗАТЕЛЬСТво. Достаточно проверить равенство

$$
\varphi(e(x), f(x))=\varphi\left(a_{0}-e_{1}(x), f_{1}(x)+\left(l_{0}-2\right) e_{1}(x)-a_{1}+a_{0}\right)
$$

и воспользоваться доказанной в лемме 1 единственностью функций $e(x)$ и $f(x)$. Лемма доказана.

Лемма 8. Целое число $x$, не делящееся на $a_{0}$, представимо через $a_{0}, a_{-1}, \ldots, a_{-n}$ тогда и только тогда, когда

$$
a_{0} \varepsilon_{2}-a_{1}+e(x)\left(1-\varepsilon_{2}\right)-f(x) \leqslant 0 .
$$

ДокАЗАТЕЛЬСТво. В полной аналогии с результатами лемм 4 и 5 число $x$ тогда и только тогда представимо через $a_{0}, a_{-1}, \ldots, a_{-n}$, когда $\left(1-\varepsilon_{1}\right) e_{1}(x)-f_{1}(x) \leqslant 0$. Поэтому для доказательства леммы достаточно подставить в последнее неравенство выражения для $e_{1}(x)$ и $f_{1}(x)$, найденные из леммы 7. Лемма доказана.

ЛЕмма 9. Если $a_{-1}>a_{0}$ и для $\alpha \in \mathbb{R}$ выполнена оценка $0 \leqslant \alpha<a_{0}$, то справедливо равенство

$$
\max _{\substack{x \notin\left\langle a_{0}, a_{-1}, \ldots, a_{-n}\right\rangle \\ e(x) \geqslant \alpha}} x=-a_{1}\left[a_{0}-\alpha\right]-a_{0}\left[-\varepsilon_{2}\left[a_{0}-\alpha\right]\right]-a_{0} .
$$


ДокАЗАТЕЛЬСТво. В полной аналогии с результатом леммы 6 максимум из настоящей леммы должен быть равен выражению $\left[a_{0}-\alpha\right] a_{-1}-a_{0}\left[\varepsilon_{1}\left[a_{0}-\alpha\right]\right]-a_{0}$. Подставляя сюда значения $a_{-1}=l_{0} a_{0}-a_{1}$ и $\varepsilon_{1}=l_{0}-\varepsilon_{2}$, получаем требуемое. Лемма доказана.

\section{5. Доказательство теорем.}

ДокАЗАТЕЛЬСТво ТЕОРЕМЫ 1. Утверждение теоремы 1 получается подстановкой значения $\alpha=a_{0}-1$ в равенство (9). Теорема доказана.

ДОКАЗАТЕЛЬСТВО ТЕОРЕМЫ 2 . ПоЛОЖим

$$
\alpha=\frac{a_{0} \varepsilon_{2}-a_{1}}{\varepsilon_{2}-\varepsilon_{1}}
$$

и покажем, что из условий $x \not \equiv 0\left(\bmod a_{0}\right), e(x) \geqslant \alpha$ и $x \in\left\langle a_{0}, a_{1}, \ldots, a_{m}\right\rangle$ следует, что $x \in\left\langle a_{0}, a_{-1}, \ldots, a_{-n}\right\rangle$. Действительно, из леммы 4 следует, что

$$
f(x) \geqslant(1-\varepsilon) e(x)=\left(1-\varepsilon_{2}\right) e(x)+\left(\varepsilon_{2}-\varepsilon\right) e(x) \geqslant\left(1-\varepsilon_{2}\right) e(x)+a_{0} \varepsilon_{2}-a_{1},
$$

и из леммы 8 следует, что $x \in\left\langle a_{0}, a_{-1}, \ldots, a_{-n}\right\rangle$.

Аналогично доказывается, что если $e(x) \leqslant \alpha, x \neq \equiv\left(\bmod a_{0}\right)$ и $x \in\left\langle a_{0}, a_{-1}, \ldots, a_{-n}\right\rangle$, то $x \in\left\langle a_{0}, a_{1}, \ldots, a_{m}\right\rangle$.

Заметим еще, что ввиду лемм 4 и 5 число $x$, представимое через

$$
a_{-n}, a_{-n+1}, \ldots, a_{-1}, a_{0}, a_{1}, \ldots, a_{m},
$$

окажется представимым через некоторые два соседних члена последовательности (12), ввиду чего

$$
\left\langle a_{-n}, a_{-n+1}, \ldots, a_{m}\right\rangle=\left\langle a_{0}, a_{1}, \ldots, a_{m}\right\rangle \cup\left\langle a_{0}, a_{-1}, \ldots, a_{-n}\right\rangle .
$$

Теперь для доказательства теоремы 2 осталось подставить значение $\alpha$ (см. (11)) в леммы 6 и 9 и взять больший из двух вариантов. Теорема доказана.

\section{6. Следствия.}

СлЕДСТВИЕ 1. Пусть $a_{0}<a_{1}, e_{m} \geqslant a_{0}\left(\right.$ əде $\left.f_{m} / e_{m}=1-\varepsilon\right) u l_{1}=\cdots=l_{m}=l \geqslant 3$. Тогда

$$
g\left(a_{0}, a_{1}, \ldots, a_{m}\right)=a_{0} a_{1}-a_{0}-a_{1}-a_{0}\left[\frac{1}{2}\left(a_{0}-1\right)\left(l-\sqrt{l^{2}-4}\right)\right] .
$$

СлЕДСтвиЕ 2. Пусть $a, k, l \in \mathbb{N}, a \geqslant 3, k \geqslant 2, l \geqslant 2$. Тогда

$$
\begin{aligned}
g(a, a & -1, a-2, k a-2 k-1, l(k a-2 k-1)-a+2) \\
& =a\left[\frac{(k l-1)(a-1)-1}{2 k l+l-2}\right]-\left[\frac{2 k l+l-2}{k l-1}\left[\frac{(k l-1)(a-1)-1}{2 k l+l-2}\right]\right]-1 .
\end{aligned}
$$

СлЕДСТВИЕ 3. Пусть $\varepsilon=a / b, \varepsilon_{2}=c / d$ - несократимые рациональные представления введенных выше чисел $\varepsilon$ и $\varepsilon_{2}$. Если выполнены равенства $b+d=a_{0}$, $a+c=a_{1}$

$$
a_{0}=\min \left\{a_{-n}, a_{-n+1}, \ldots, a_{0}, a_{1}, \ldots, a_{m}\right\},
$$

то имеет место равенство

$$
g\left(a_{-n}, a_{-n+1}, \ldots, a_{0}, a_{1}, \ldots, a_{m}\right)=a_{m}-a_{0} .
$$

Продолжение следует. 


\section{СПИСОК ЦИТИРОВАННОЙ ЛИТЕРАТУРЫ}

[1] Sylvester J. J. Mathematical questions, with their solutions // Educational Times. 1884. V. 41. P. 21.

[2] Johnson S. M. A linear Diophantine problem // Canad. J. Math. 1960. V. 12. P. 300-398.

[3] Шевченко В.Н. Качественные вопросы целочисленного программирования. М.: Наука (Физматлит), 1995.

[4] Brauer A., Shockly J.E. On a problem of Frobenius // Angew. Math. 1962. V. 211. P. $215-220$.

[5] Roberts J. B. Note on linear forms // Proc. Amer. Math. Soc. 1956. №7. P. 465-469.

[6] Brauer A. On a problem of partitions // Amer. J. Math. 1942. V. 64. P. 299-312.

[7] Siering E. Über lineare Formen und ein Problem von Frobenius // J. Reine Angew. Math. 1974. V. 271. P. 177-202.

[8] Rödset Ö. J. On a linear Diophantine problem of Frobenius-II // J. Reine Angew. Math. 1979. V. 307/308. P. 431-440.

[9] Кан И. Д., Стечкин Б. С., Шарков И. В. К проблеме Фробениуса трех аргументов // Матем. заметки. 1997. Т. 62. № 4. С. 626-629.

[10] Кан И. Д. Рекуррентные последовательности и их приложения. Дисс. ‥ д.ф.-м.н. М.: МГУ, 1997.

[11] Кан И.Д. К проблеме Фробениуса // Фундамент. и прикл. матем. 1997. Т.3. № 3. C. 821-835.

[12] Финкельштейн Ю. Ю. Односторонние наилучшие приближения и их геометрическая интерпретация // Экономика и матем. методы (Москва). 1992. Т. 28. № 1. С. 130-136. 